\title{
Formation of Anisotropic Polymer Colloids by Disparate Relaxation Times
}

\author{
Willem K. Kegel, ${ }^{*} \dagger$ Dana Breed, Mark Elsesser, and David J. Pine \\ Center for Soft Matter Research, Department of Physics, New York University, 4-6 Washington Place, \\ New York, New York 10003
}

Received June 21, 2006

\begin{abstract}
We show that coupling between a fast and a slow relaxation time causes the spontaneous formation of protrusions in colloids made of cross-linked polymers. The volume of the protrusions can be controlled by adjusting the ratio between the relaxation times. This, in principle, results in particles with levels of anisotropy that can be made "to order".
\end{abstract}

Anisotropic colloidal particles may in principle form crystal structures that are completely different from their isotropic analogues. Under certain conditions, giant micelles or shell-like superstructures are expected. These particles therefore are of immense interest for fundamental study as well as for potential applications. Much progress has recently been made in methodologies to prepare anisotropic particles consisting of heterogeneous polymers. Resulting particles vary in shape from "snowman/confetti" to dented ${ }^{2}$ and egglike, ${ }^{3}$ just to mention a few.

Sheu and co-workers ${ }^{4,5}$ made use of interpenetrating homopolymer networks of polystyrene to produce spherical crosslinked polystyrene particles with protrusions of both cross-linked and non-cross-linked polystyrene. Protrusions are formed upon swelling of cross-linked polystyrene particles with styrene, followed by heating. Heating causes the network to shrink and expel excess styrene in the form of protrusions and at the same time results in the polymerization of styrene monomers. The above method works only if the seed particles are at least several micrometers in size. Significant progress has very recently been made by modification of the surface of the seed particles, resulting in anisotropic particles with sizes on the order of $100 \mathrm{~nm}^{6}$

An intriguing observation ${ }^{4}$ is the spontaneous formation of protrusions upon swelling of the particles with styrene, even without heating. This observation opens up the possibility to prepare particles with protrusions that contain material that is unstable to heating, such as fluorophores, proteins, and other biomolecules, making them in principle relevant for applications such as controlled release and artificial drug and gene delivery. Indeed, by being able to control the formation of protrusions, the relevant materials may be ejected from the polymer networks on the spot and at the right time. Here we show that the mechanism of the spontaneous formation of protrusions upon swelling is coupling between two disparate relaxation times, a fast one coupled to swelling of the network and a slow one connected to shrinking. We show that the anisotropy of the particles can be modified by systematically varying the relaxation times.

\footnotetext{
* Corresponding author. E-mail: w.k.kegel@chem.uu.nl.

$\dagger$ On leave from Van't Hoff Laboratory, Utrecht University, Padualaan 8, $3584 \mathrm{CH}$ Utrecht, The Netherlands.

(1) Okubo, M.; Fujibayashi, T.; Yamada, M.; Minami, H. Colloid Polym. Sci. 2005, 283, 1041-1045.

(2) Okubo, M.; Saito, N.; Fujibayashi, T. Colloid Polym. Sci. 2005, 283, 691698.

(3) Ni, H.; Ma, G.; Nagai, M.; Omi, S. J. Appl. Polym. Sci. 2001, 80, 20022017.

(4) Sheu, H. R.; El-Aasser, M. S.; Vanderhoff, J. W. J. Polym. Sci., Part A. Polym. Chem. 1990, 18, 629-651.

(5) Sheu, H. R.; El-Aasser, M. S.; Vanderhoff, J. W. J. Polym. Sci., Part A Polym. Chem. 1990, 18, 653-667.

(6) Mock, E. B.; DeBruyn, H.; Hawkett, B. S.; Gilbert, R. G.; Zukoski, C. F. Langmuir 2006, 22, 4037-4043.
}

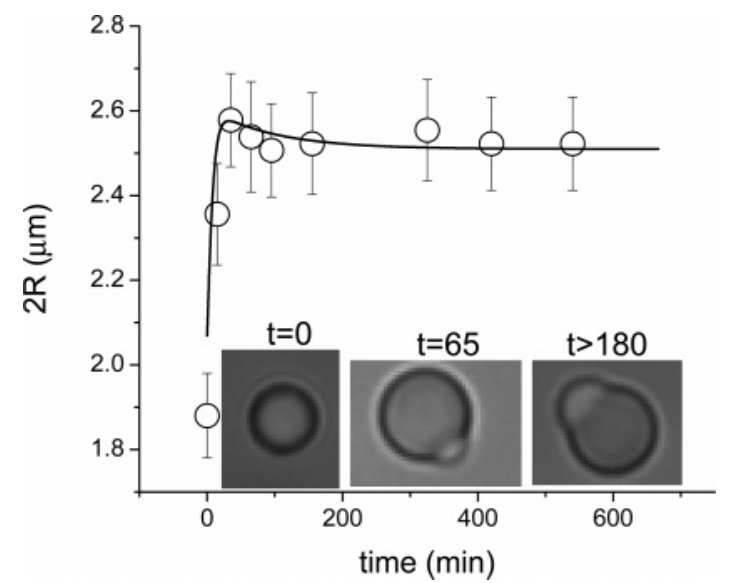

Figure 1. Average diameter $2 R$ of the cross-linked polystyrene particles as a function of time after adding styrene to the mixture. Points, experiments; line, theory (eq 2 with eq 1 ) with $2 R_{0}=1.88$ $\mu \mathrm{m}, \tau_{\mathrm{f}}=6 \mathrm{~min}, \tau_{\mathrm{s}}=100 \mathrm{~min}, \alpha=0.08$, and $\phi^{\infty}=0.58$. The inset shows representative snapshots of single spheres after 0,65 , and 180 min as indicated.

Our experimental setup is as follows: $4.5 \mathrm{~mL}$ of an aqueous dispersion of 3\% (w/w) Magsphere polystyrene particles of 1.88 $\mu \mathrm{m}$ diameter, cross linked with $5 \%$ divinylbenzene and containing $1 \%(\mathrm{w} / \mathrm{w}) \mathrm{SDS}$, was mixed with $1.1 \mathrm{~g}$ of styrene. The mixture was gently stirred at $20^{\circ} \mathrm{C}$, and samples were taken at several time intervals and inspected under a light microscope.

In Figure 1, the diameter of the primary part of the particles (i.e., not including the protrusions) has been plotted as a function of time. The primary particles first swell with styrene and then slowly deswell, consistent with the expulsion of excess styrene in the form of a protrusion.

We model the initial swelling, which occurs on a time scale $\tau_{\mathrm{f}}$ much faster than the subsequent deswelling, as a simple exponential: $\phi(t)=\phi^{*}\left(1-\mathrm{e}^{-t / \tau_{\mathrm{f}}}\right)$. Here, $\phi^{*}$ is the equilibrium styrene concentration if swelling were the only process to occur. If during swelling, however, $\phi^{*}$ changes in time as a result of some slow process (as yet unidentified) that is triggered by the swelling, then more complex behavior may be expected. To allow for this possibility, we suppose that the styrene concentration $\phi^{*}$ evolves exponentially toward some ultimate volume fraction $\phi^{\infty}$ with a slow time constant $\tau_{\mathrm{s}} \gg \tau_{\mathrm{f}}$. Combining this with the fast swelling process, we obtain the following model for the time-dependent volume fraction of styrene within the swollen particle:

$$
\phi(t)=\phi^{\infty}\left(1-e^{-t / \tau_{\mathrm{f}}}\right)\left(1+\alpha \mathrm{e}^{-t / \tau_{\mathrm{s}}}\right)
$$


The time-dependent radius of the particles immediately follows from eq 1 as

$$
R(t)=R_{0}(1-\phi(t))^{-1 / 3}
$$

where $R_{0}$ denotes the radius of the unswollen particle.

In general, a maximum in the particle size as a function of time is found if $\alpha>0$ and $\tau_{2} \gg \tau_{1}$. In "fitting" the data to eqs 1 and 2 , we set $\alpha=0.1$, this being the estimated relative volume of the protrusions after more than $24 \mathrm{~h}$ since the start of the experiment. Because swelling occurs within minutes, we let the fast relaxation time vary between 1 and $10 \mathrm{~min}$, and because the first sign of protrusions appeared an hour after the start of the experiment, we let the slow relaxation time vary between 10 and 100 minutes. The final values corresponding to the best agreement with experiments are given in the caption of Figure 1.

The fast relaxation time $\tau_{\mathrm{f}}=6 \mathrm{~min}$ is not compatible with swelling being limited by the diffusion of styrene in water. The diffusion coefficient of styrene $D_{\mathrm{s}} \approx 10^{-9} \mathrm{~m}^{2} / \mathrm{s}$, which corresponds to a characteristic diffusion time in the particles of $R^{2} / D_{\mathrm{s}} \approx 0.001$ $\mathrm{s}$, which is much too small. Therefore we conclude, as in refs 7 and 8 , that the relaxation of the initially glassy polymer network is the time-limiting step in the swelling of the particles.

However, we observe an even slower time scale, associated with the shrinking of the particles and the consequent formation of protrusions. This time scale probably is related to the (very) slow formation of cross links by unreacted ends and cross linkers in the polymer molecules in the devitrifying network. Note that unreacted cross linkers have not been removed and will be mobilized upon swelling and devitrification of the network. We note that swelling and subsequent shrinking of cross-linked polymer networks have also been observed in the sorption of gaseous methanol by PVC, ${ }^{7}$ pointing to a quite general effect in cross-linked polymer networks. In ref 6 , the surface of crosslinked polystyrene was made hydrophilic, resulting in increased asphericity of the expelled monomer bulge (and of the final particle) caused by a modified wetting angle between the expelled monomer and seed particle. This procedure led to a significant improvement of the original work of Sheu and co-workers, ${ }^{4,5}$ which was limited to relatively large particles several micrometers in size. Being able to modify the volume of the protrusions opens up the possibility to adjust the anisotropy of the resulting colloidal particles "to order". In principle, modification of the ratio between the relaxation times coupled to swelling and deswelling can modify the volume of the protrusion formed. This is corroborated by Figure 2, where it can be seen that the maximum in the particle size, being a measure of the size of the resulting protrusion, is predicted to become smaller and disappears when the relaxation times become comparable. Note that increasing size and crosslinking density of the particles will increase the disparity between the slow and fast relaxation times, leading to larger protrusions. This, indeed, has been observed experimentally. ${ }^{4}$

We choose to test the scenario discussed above and depicted in Figure 2 by making use of the wide variation in time scales provided by diffusion. In Figure 3, the experimental setup is shown schematically. A layer of styrene (solubility in water $\approx$ $0.3 \mathrm{~g} / \mathrm{L}$ ) was gently added to an aqueous colloidal suspension as described above in a Vitrocom $0.05 \times 1.0 \mathrm{~mm}^{2}$ capillary and subsequently sealed and glued onto an object slide using Norland 81 optical adhesive UV glue. In this setup, the distance $L$ from a colloidal particle to the interface between the colloidal suspension and styrene defines the diffusion time of styrene, which is given by $L^{2} / 6 D_{\text {s }}$. At the distance $L$ where $L^{2} / 6 D_{\text {s }}$ becomes

(7) Berens, A. R.; Hopfenberg, H. B. Polymer 1978, 19, 489-496.

(8) Zeeuw, E. A. v. d.; Sagis, L. M. C.; Koper, G. J. M. Macromolecules 1996, $29,801-803$.

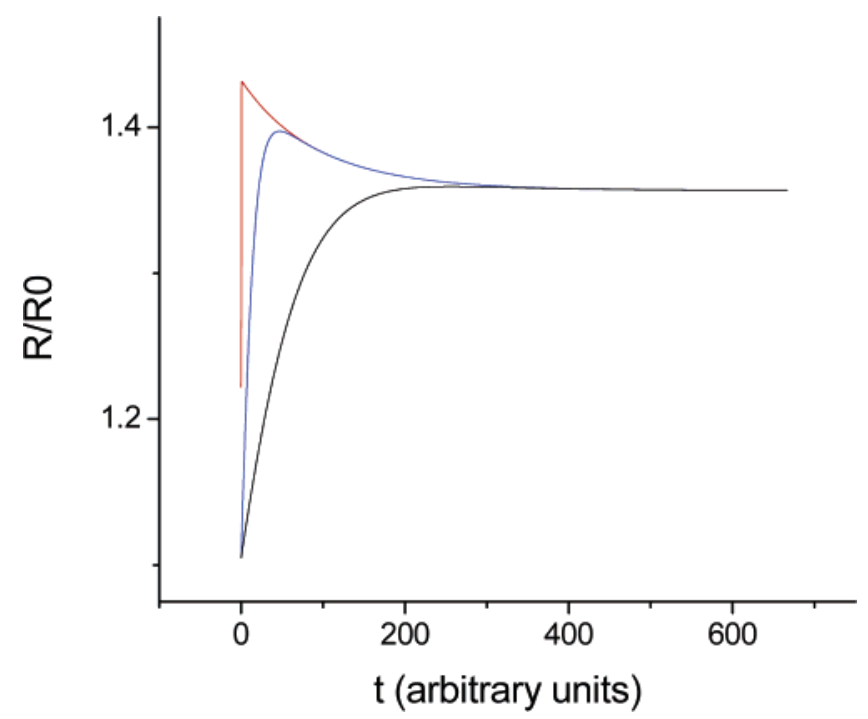

Figure 2. Evolution of average radius in time, eqs 1 and 2, with a fixed slow time scale $\tau_{\mathrm{s}}=100$ and varying $\tau_{\mathrm{f}}=1$ (red), 100 (blue), and 500 (black).

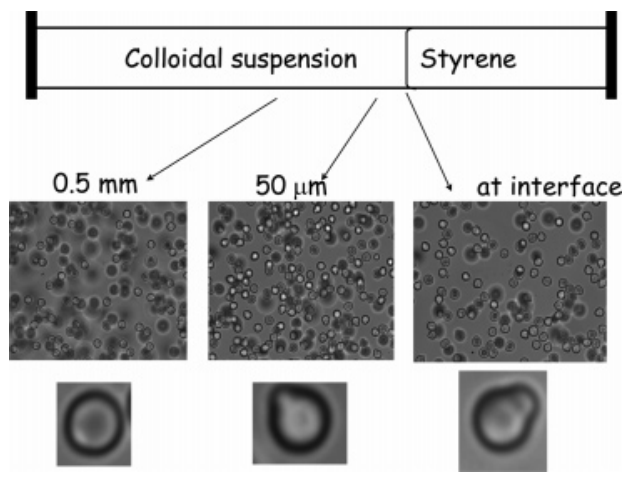

Figure 3. Snapshots of cross-linked polystyrene particles in a capillary at several distances $L$ from the interface with styrene as schematically indicated. Pictures were taken $24 \mathrm{~h}$ after the preparation of the capillary. See the text.

comparable to the slow relaxation time $\tau_{\mathrm{s}}$, we expect protrusions to disappear, as illustrated in Figure 2. At $L=50 \mu \mathrm{m}$ from the interface (Figure 3), the typical diffusion time of styrene is 0.42 $\mathrm{s}$, which is much shorter than the slow relaxation time $\tau_{\mathrm{s}}$. At $L$ $=0.5 \mathrm{~mm}$, it is $1.16 \mathrm{~h}$, which is comparable to the slow relaxation in the system and much slower than the fast relaxation time $\tau_{\mathrm{f}}$, so that the diffusion of styrene to the particles, and not the relaxation of the network, becomes the limiting time scale for the swelling of the particles. It is between these two distances where a crossover is expected. This is indeed what we observe: $24 \mathrm{~h}$ after preparing the capillary, at $L=50 \mu \mathrm{m}$ there are still protrusions, comparable to the situation closer to the interface. At $L=0.5 \mathrm{~mm}$, the protrusions have almost disappeared. Note that the typical diffusion time for the colloids over $0.5 \mathrm{~mm}$ is more than 2 days, the diffusion coefficient of a $1 \mu \mathrm{m}$ spherical particle in water being $2.1 \times 10^{-13} \mathrm{~m}^{2} / \mathrm{s}$. Therefore, in the time window in which the aqueous dispersion is saturated with styrene, the colloids have hardly moved.

In conclusion, two disparate time scales are responsible for the formation of protrusions in cross-linked polymer colloids at constant temperature. The anisotropy of the particles can be controlled by adjusting the time scales.

Acknowledgment. We thank Andy Hollingsworth for help with experiments and Alfons van Blaaderen and Jan Groenewold for discussions.

LA061792Z 